\title{
Synthesis of Nanocrystalline Electron Emissive Materials with Homogenous Composition in Nanoscale
}

\author{
M. Shiran, M. J. Hadianfard, and M. M. Shiezadeh
}

\begin{abstract}
In this research, specific stoichiometric mixture of nanocrystalline barium carbonate, calcium carbonate, and aluminum oxide was fabricated by using the sol-gel combustion method. This mixture is used as emissive materials on impregnated cathodes which make up high power vacuum microwave tubes such as klystrons. Composition uniformity and particle size of emissive materials affect the electron emission properties of impregnated cathodes. In this study, dry gel and heat treated powder at $500{ }^{\circ} \mathrm{C}$ are investigated by thermal analysis (TG/DTA), X-ray diffraction (XRD), scanning electron microscopy (SEM), and energy dispersive $X$-ray spectroscopy (EDX). The results show that dry gel combusts at $260{ }^{\circ} \mathrm{C}$, and that during combustion transforms from an amorphous phase to a nanocrystalline phase. The average crystallite size of the powders which is calculated by Debye-Scherrer's method based on the XRD patterns is under $45 \mathrm{~nm}$. The results of SEM and EDX of powder after heat treatment at $500{ }^{\circ} \mathrm{C}$ show that the particle size of powder is under $100 \mathrm{~nm}$, and the chemical composition of emissive material is uniform at nanoscale.
\end{abstract}

Index Terms-Dispenser cathode, emissive material, nanopowder, sol-gel combustion synthesis.

\section{INTRODUCTION}

Thermionic dispenser cathodes are widely used in fabricating electron beam devices due to the production of high current density at low temperature. In order to reduce the work function of the dispenser cathode surface, the emissive materials, which are barium-calcium aluminates, are diffused into tungsten pellet pores [1]-[6]. The specific molar ratio of barium oxide, calcium oxide, and aluminum oxide ( $\mathrm{BaO}: \mathrm{CaO}: \mathrm{Al}_{2} \mathrm{O}_{3}$ ) is 5:3:2. This is blended to use as an emissive material for impregnating dispenser cathodes [1]. It is normal to use emissive materials in the form of barium carbonate, calcium carbonate, and aluminum oxide mixture because carbonate mixture is more resistant to the absorption of moisture than an oxide mixture. Normally, ball-milling method is used to obtain a uniform blend of emissive material [7]. The disadvantage of using ball-milling method is that the probability of entering impurities into the emissive materials is high. Alternatively, sol-gel method is a suitable way to obtain a homogenous mixture with the lowest amount of impurities. Moreover, using sol-gel technique instead of ball-milling method for the fabrication of cathode enhances the emission properties of it. Wang et al. [8] compared the emission properties of scandate cathodes fabricated by sol-gel

Manuscript received April 1, 2013; revised May 30, 2013.

The authors are with the School of Materials Science and Engineering, Shiraz University, Shiraz, Iran (tel.: 0098-0913-303-6055; e-mail m.shiran01@gmail.com, hadianfa@shirazu.ac.ir, hgold86@gmail.com). technique and ball-milling method. They reported that the emission properties of cathode synthesized by sol-gel technique were better than that of cathode produced through ball-milling method. They claimed that small particles and more uniform mixing bring about good emission capability of scandate cathode synthesized by sol-gel technique.

Generally, the sol-gel combustion method is a novel technique for the production of crystalline and high purity powders. Sol-gel combustion technique is based on a chemical sol-gel and the subsequent combustion process. During the sol-gel process, an aqueous solution containing salts of the desired metal and an organic fuel form a gel. After the gel obtained, ignition occurs in the gel due to combustion process. The product of this step is voluminous and fluffy with a large surface area. This technique is characterized by inexpensive raw materials, low temperature synthesis, and obtaining nanocrystalline powder with homogenous composition [9]. Studies have shown that using emissive materials with uniform composition and crystallite size at nanoscale improve emission performance of cathode. Wang et al. [10] fabricated oxide cathode using nano emissive materials which is produced by liquid-gas method. They reported that the emission current density of oxide cathode used nano emissive materials is significantly higher than that of the conventional oxide cathode under the same condition.

The main goals of this work were to synthesize appropriate mixture of emissive materials by sol-gel combustion technique and investigation of crystalline structure and the composition uniformity of synthesized powder.

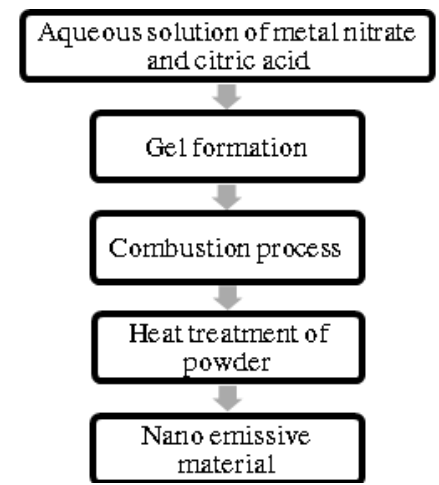

Fig. 1. Process for synthesis of nano emissive material powder.

\section{EXPERIMENTAL METHODS}

Nanocrystalline emissive material with the molar ratio of 5:3:2 was prepared by sol-gel combustion technique. The nanocrystalline emissive material fabrication-process sequence is illustrated in Fig. 1. According to Fig. 1, the appropriate amount of barium nitrate $\left(\mathrm{Ba}\left(\mathrm{NO}_{3}\right)_{2}\right.$, Merck), 
calcium nitrate $\left(\mathrm{Ca}\left(\mathrm{NO}_{3}\right)_{2} \cdot 4 \mathrm{H}_{2} \mathrm{O}\right.$, Merck), aluminum nitrate $\left(\mathrm{Al}\left(\mathrm{NO}_{3}\right)_{3} .9 \mathrm{H}_{2} \mathrm{O}\right.$, Merck) and citric acid $\left(\mathrm{C}_{6} \mathrm{H}_{8} \mathrm{O}_{7} \cdot \mathrm{H}_{2} \mathrm{O}\right.$, Merck) were first dissolved in distilled water. The molar amount of citric acid was three times more than the total molar amount of metal nitrates in solution. Then the solution was heated at $60{ }^{\circ} \mathrm{C}$ and stirred continuously for $20 \mathrm{~h}$ to obtain

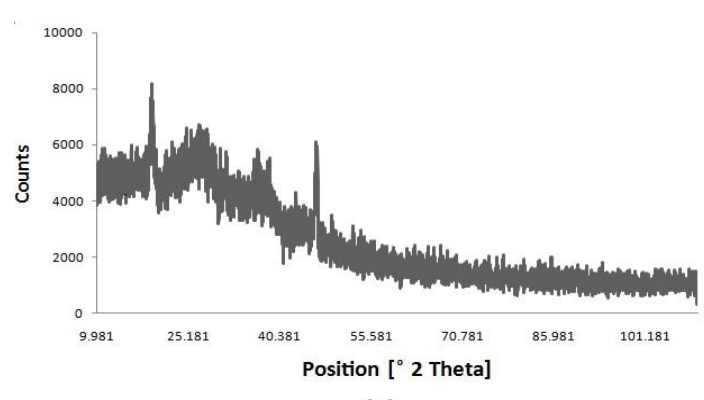

(a) uniform sol. Afterwards, the stabilized nitrate-citrate sol was heated at $100{ }^{\circ} \mathrm{C}$ to obtain a porous dry gel. The dry gel automatically gets ignited in air at $260{ }^{\circ} \mathrm{C}$ as a result of thermally induced redox reaction. The method of ignition was auto-combustion which started at a point and developed throughout the gel.

Fig. 2. (a) XRD pattern of the dry gel and. (b) SEM image of the dry gel

After combustion process, black soft powder was produced. To remove residual organic materials, the sample was heat treated at $500^{\circ} \mathrm{C}$ for $2 \mathrm{~h}$ and nano emissive materials were produced.

Thermal decomposition of the dried gel was investigated by thermal gravimetric analysis and differential thermal analysis (DTA/TG, PL-STA-1640) at a heating rate of $5{ }^{\circ} \mathrm{C} / \mathrm{min}$ from $25^{\circ} \mathrm{C}$ to $900{ }^{\circ} \mathrm{C}$ in air. Phase identification of the dry gel and investigation of both the phase and crystalline structure of the heat treated powder were investigated by X-ray diffractometer (Shimadzu X-pert Diffraction, Lab $\mathrm{X}-6000)$ with $\mathrm{Cu} \mathrm{K}_{\alpha}$ radiation $(\lambda=1.5405 \AA)$. Morphology, particle shape, and the composition uniformity of the heat treated powder and dry gel were characterized by scanning electron microscopy and energy dispersive spectroscopy (SEM/EDX, Carl Ziess Merilin).

\section{RESULTS AND DISCUSSION}

Fig. 2 shows both the XRD pattern of the dry gel before combustion and the related SEM image. As shown in Fig 2(a), no significant crystalline phase was observed in the XRD pattern of the dry gel. It means that the dry gel has consisted of an amorphous phase before combustion. Absence of any significant crystalline phase is due to the distribution of metallic ions uniformly throughout the gel in atomic scale. This amorphous phase restricts metal ions from moving throughout the gel and from forming crystalline phases [11], [12]. During the gel drying process, keeping metallic ions apart by a gel network causes the dry gel to become almost amorphous. The microstructure of the dry gel before combustion process is shown in Fig. 2(b). A continuous phase with a porous microstructure was observed due to cohesive properties of the gel and the release of $\mathrm{NH}_{3}$ and $\mathrm{H}_{2} \mathrm{O}$ gases during the drying process.

Fig. 3 presents the TG/DTA curves of the dry gel. The DTA plot shows two exothermic peaks. In Fig. 3, the first exothermic peak, appeared at about $260{ }^{\circ} \mathrm{C}$, could be due to the process of combustion in the gel. Combustion process is a redox reaction of the gel wherein citrate ions act as reductant and nitrate ions act as oxidant. During redox reaction throughout the gel, large amounts of gas are released that contain $\mathrm{H}_{2} \mathrm{O}, \mathrm{CO}_{2}$ and $\mathrm{N}_{2}$. Releasing these gases led to a noticeable weight loss $(40 \%)$ in TG plot. The second exothermic peak in DTA plot at $490{ }^{\circ} \mathrm{C}$ was attributed to the decomposition of residual citric acid led to $25 \%$ weight loss in TG plot.

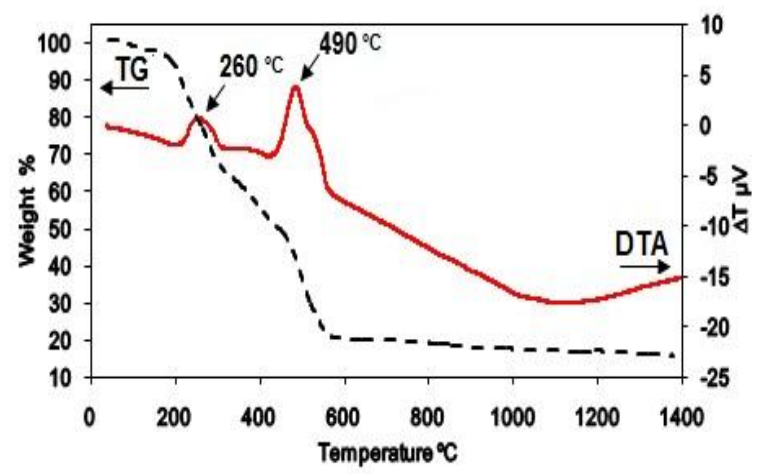

Fig. 3. DTA/TG traces of the dried gel

Generally, the thermal analysis of an exothermic reaction in a sol-gel combustion process shows one dramatic exothermic peak. A rapid reduction in weight during combustion shown in TG graph also occurs in one step [10], [12], [13]. It is also observed that there are two exothermic peaks in the thermal analysis of some combustion processes which are wider and weaker than the only peak in the common processes. Li et al. [9] reported that the nature of sol-gel combustion and the molar ratio of citric acid to metal nitrates $(\mathrm{CA} / \mathrm{MN})$ are the main causes of this difference. In addition, the position and intensity of two exothermic peaks shown by TG/DTA are changed by increasing the CA/MN molar ratio [10]. We can conclude that the existence of two exothermic peaks in DTA plot in Fig. 3 was related to high molar ratio of CA/MN.

The result of the XRD analysis of the powder which was heat treated at $500{ }^{\circ} \mathrm{C}$ for $2 \mathrm{~h}$ is shown in Fig 4. Investigation of the XRD pattern of the heat treated powder showed the presence of crystalline phases relating to barium carbonate, calcium carbonate and aluminum oxide. The presence of crystalline phases depended on the redox reaction in the dry gel during combustion. The reaction of citric acid with $\mathrm{Ba}^{2+}$ 
and $\mathrm{Ca}^{2+}$ ions which were homogeneously distributed throughout the polymeric citric acid matrix caused the creation of a "metallic ion-citric acid" complex. The precipitated barium carbonate and calcium carbonate appeared due to the redox reaction between metallic ions and the disconnected-carboxyl groups of citric acid. Moreover, during combustion, the redox reaction among $\mathrm{Al}^{3+}$ ions and carboxyl groups of citric acid led to the creation of $\mathrm{Al}_{2} \mathrm{O}_{3}$.

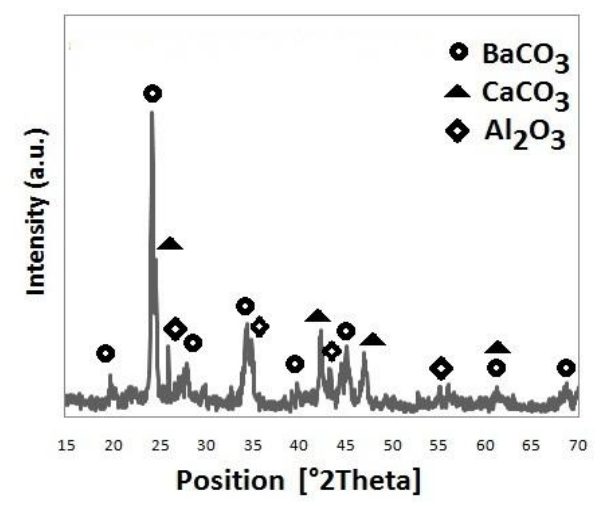

Fig. 4. XRD pattern of heat treated powder at $500^{\circ} \mathrm{C}$.

The average crystallite size of the emissive powder was calculated by Debye-Scherrer's method (1) based on the XRD pattern [11], [13].

$$
D=k \lambda / \beta \cos \theta
$$

where $D$ is the crystallite size in $\mathrm{nm}, \lambda$ is the radiation wavelength $(0.15405 \mathrm{~nm}), \beta$ is the half width of maximum peak, $\theta$ is the diffraction peak angle, and $k$ is a shape factor taken as 0.89 . As can be seen in Fig. 4, barium carbonate reached a maximum peak at $\theta=12.0328 \mathrm{deg}$ with $\beta=0.246 \mathrm{deg}$. Crystallite size of barium carbonate was calculated around 41 $\mathrm{nm}$ by using (1). Table I gives some crystal planes and crystallite size of barium carbonate, calcium carbonate and aluminum oxide. Crystallite size of emissive powder was calculated by X'Pert HighScore software and Scherrer's equation according to the characteristics of the emissive powders X-ray pattern. As can be seen in Table I, the crystallite size of emissive powder is less than $45 \mathrm{~nm}$. During combustion, there is not enough time and energy for defusing atoms at long atomic distance owing to the uniform distribution of reactants in gel network at nanoscale and the rapid combustion reaction, hence crystallite size remained constant. Through heat treatment of the emissive powder, there was a growth in crystallite size, though it was not noticeable.

Fig. 5 shows the SEM images of the heated treated emissive powder and the related EDX results. According to Fig. 5(a), after heat treatment of the powder, emissive material particles with sizes below than $100 \mathrm{~nm}$ formed a porous and voluminous structure with irregular morphology. In this structure, agglomeration between nanometer sized particles took place which formed a large network with irregular size and shape. This was due to a vigorous redox reaction occurring during combustion, which released a large amount of gas due to the thermal decomposition of citric acid.

TABLE I: THE XRD PARAMETER AND CRYSTALLITE SIZE OF THE EMISSIVE

\begin{tabular}{cccccc}
\hline \multicolumn{7}{c}{ MATERIAL COMPONENTS. } \\
\hline \hline Composition & hkl & $2 \theta$ & $\begin{array}{c}\text { Relative } \\
\text { Intensity } \\
(\%)\end{array}$ & $\begin{array}{c}\text { FWHM } \\
2 \theta\end{array}$ & $\begin{array}{c}\mathrm{D} \\
(\mathrm{nm})\end{array}$ \\
\hline BaCO $_{3}$ & 111 & 24.0656 & 100 & 0.1966 & 40.86 \\
$\mathbf{B a C O}_{3}$ & 110 & 19.7526 & 5.40 & 0.5716 & 13.96 \\
$\mathbf{C a C O}_{3}$ & 100 & 24.4922 & 48.57 & 0.1963 & 40.98 \\
$\mathbf{C a C O}_{3}$ & 004 & 42.2286 & 28.98 & 0.246 & 34.26 \\
$\mathbf{A l}_{\mathbf{2}} \mathbf{O}_{3}$ & 110 & 25.84 & 11.43 & 0.2952 & 27.32 \\
$\mathbf{A l}_{\mathbf{2}} \mathbf{O}_{3}$ & 121 & 34.822 & 22.24 & 0.2952 & 27.91 \\
\hline \hline
\end{tabular}

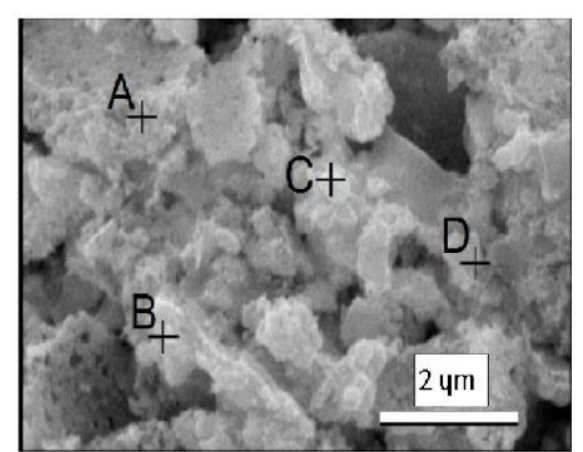

(a)
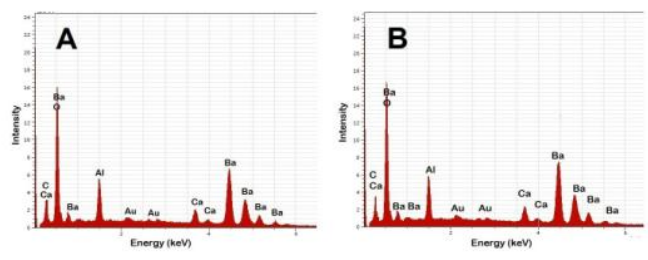

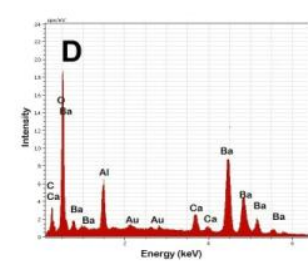

(b)

Fig. 5. (a) SEM image and (b) EDX results of heat treated powder of nano emissive materials at A, B, C, and D points.

In Fig. 5(b), the EDX analysis results from A, B, C, and D points on the SEM image of the heat treated powder indicate the existence of barium, calcium and aluminum elements at every four points. It can be concluded from the comparison of the intensities of element peaks at each $\mathrm{A}, \mathrm{B}, \mathrm{C}$, and D point that the concentration of each element at four points is equal; therefore, the chemical composition of the powder is uniform at nanoscale. This homogeneity is caused by the nature of sol-gel combustion. The carboxyl gropes in sol increase by using a high molar ratio of $\mathrm{CA} / \mathrm{MN}$ in the starting solution; 
hence, there are a lot of "metallic ion-citric acid" complexes in the gel, which homogenously distribute throughout the gel. This led to significant improvement in composition homogeneity of emissive powder after combustion.

Using nano emissive materials with homogenous composition at nanoscale improves the emission uniformity of cathode through producing free $\mathrm{Ba}$ and $\mathrm{BaO}$ homogeneously. During the activation process of the cathode, releasing barium and barium oxide uniformly leads to the formation of homogenous Ba-O layer on the cathode surface. This results in achieving a uniform electron emission from the surface of the cathode. Also, we observed that nano emissive materials melted at lower temperature about $100{ }^{\circ} \mathrm{C}$ in comparison with melting point of conventional emissive materials under the same condition. Reducing melting point of nano emissive material preserves the cathode against forming undesirable phases during the impregnation process of tungsten pellet.

\section{CONCLUSION}

Electron emissive nanocrystalline powder in the form of initial barium carbonate, calcium carbonate and aluminum oxide mixture have been synthesized by the sol-gel combustion method using corresponding metal nitrates and citric acid. A high rate and low energy-level redox reaction led to the formation of barium carbonate, calcium carbonate and aluminum oxide crystals with crystallite size under $45 \mathrm{~nm}$. The emissive material powder produced by sol-gel combustion method had chemical homogeneity, and the components were mixed together at molecular-level. Reducing crystallite and particle size of emissive powder makes it chemically active to react with tungsten pellet during activation process. This leads to an increase in the tendency to produce free $\mathrm{Ba}$ and $\mathrm{BaO}$. In addition, improvement in the composition homogeneity of nano emissive material causes uniformly to release $\mathrm{Ba}$ and $\mathrm{BaO}$. We believe that the cathode impregnated by this nano emissive material can show excellent emission properties.

\section{REFERENCES}

[1] J. L. Cronin, "Modern dispenser cathodes," IEE Proc, vol. 128, pp19-32, Feb. 1981.
[2] S. Yamamoto, "Fundamental physics of vacuum electron sources," Rep. Prog. phys, vol. 69, pp. 181-232, Jan. 2006.

[3] S. Kimura, k. Homma, M. Nikaido, K. Kobayashi, T. Higuchi, and Y. Ouchi, "Long-life high-reliability Ir-coated dispenser cathode," International Electron Devices Meeting, 1987, pp. 689-692.

[4] D. Month and L. Thornburg, "Method of fabricating of porous tungsten body for a dispenser cathode," U.S. Patent 3653 883, April 1, 1970.

[5] T. Higuchi, S. Matsumoto, K. Koyama, A. Hara, and H. Hamamoto, "Life estimation of Ir-coated dispenser cathodes and heaters for," Applied Surface Science, vol. 146, pp. 109-116, May. 1999.

[6] B. K. Vancil, R. A. Mueller, E. R. Steele, and W. L. Ohlinger, "The metallurgical properties of tungsten-iridium cathodes," Applied Surface Science, vol. 146, pp. 39-46, May. 1999.

[7] J. Wang, X. Zhang, W. Liu, Y. Cui, Y. Wang, and M. Zhou, "Preparation and emission property of scandia pressed cathode," Journal of rare earths, vol. 28, pp. 460-463, 2010.

[8] J. Wang, W. Liu, L. Li, Y. Wang, Y. Wang, and M. Zhou, "A study of scandia-doped pressed cathodes," IEEE Transaction on Electron Devices, vol. 56, pp. 799-804, May. 2009.

[9] Y. Li, L. Xue, L. Fan, and Y. Yan, "The effect of citric acid to metal nitrates molar ratio on sol-gel combustion synthesis of nanocrystalline LaMnO3 powders," Journal of Alloys and Compounds, vol. 478, pp. 493-497, Jun. 2009

[10] Q. F. Wang, X. X. Wang, and J. R. Luo, "Research on the nano-particle carbonates oxide cathode," International Vacuum Electron Sources Conference and Nanocarbon, 2010, pp. 45-46.

[11] A. Zelati, A. Amirabadizadeh, and A. Kompany, "Preparation and characterization of barium carbonate nanoparticles," International Journal of Chemical Engineering and Applications, vol. 2, pp. 299-303, Aug. 2011.

[12] A. Mali and A. Ataie, "Structural characterization of nano-crystalline $\mathrm{BaFe}_{12} \mathrm{O}_{19}$ powders synthesized by sol-gel combustion route," Scripta Materialia, vol. 53, pp. 1065-1070, Nov. 2005.

[13] S. A. Seyyed Ebrahimi and J. Azadmanjiri, "Evaluation of NiFe2O4 ferrite nanocrystalline powder synthesized by a sol-gel auto-combustion method," Journal of Non-Crystalline Solids, vol. 353, pp. 802-804, April. 2007.

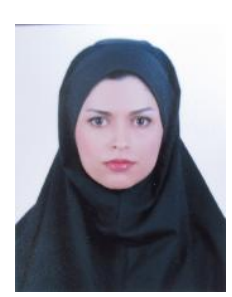

Mina Shiran was born in Isfahan, Iran, on September 21, 1986. She received the B.S. degree from Azad University of Maybod, Yazd, Iran, and M.S. degree in 2012 from Shiraz University, Shiraz, Iran, with the dissertation "Fabrication of Thermionic Cathode Using Nanotechnology and Failure Analysis of $\mathrm{M}$ Type Impregnated-Dispenser-Cathode."

She worked at Shiraz Industry for 2 years as a researcher. Her current interests include nanocomposite materials, thermionic cathode, especially scandate pressed cathodes. 